gen, die Sucht, staatlich und privat Aufwand und Prunk als Selbstzweck vorzuführen. Das aztekische Reich wird daher von D. als "Potlatch-Staat“ (S. 259) eingeordnet, potlatch als Hang, mit allen Kräften vorübergehend Reichtum mit dem alleinigen $Z$ wecke anzuhäufen, alles bei einer Gelegenheit zu verschenken. Derartige Staatsfeierlichkeiten zwecks Beeindruckung noch unbesiegter Völker werden aus Anlaß der offiziellen Krönungsfeiern geschildert (S. 208-210): An einem einzigen Tag wurden bei der Krönung von Ahuítzotl Geschenke verteilt, die dem jährlichen Tribut entsprachen, der nach Tenochtitlan floß. Oder anders ausgedrückt: Der Umfang der Geschenke entsprach etwa dem, als hätte man bei der Krönung Eduards VII. die gesamten Staatseinkünfte des britischen Imperiums einschließlich aller in Indien gefundenen Edelsteine verschenkt. Die Azteken, sie waren ein außergewöhnliches Staatsvolk.

Gerhard Scheffler

\title{
Michael Domitra
}

Die Rolle der Gewerkschaften im mexikanischen Herrschaftssystem

Verlag Neue Gesellschaft GmbH, Bonn-Bad Godesberg 1975, S. 312.

Eine organisierte Arbeiterschaft in Entwicklungsländern hat auf der Hand liegende allgemeinpolitische und ökonomische Bedeutung für die Modernisierung dieser Staaten. Deren Gesellschaft ist in der Regel durch das weitgehende Fehlen einer strukturellen Verflechtung der verschiedenen Gesellschaftsgruppen gekennzeichnet mit der Folge, daß sich nur langsam eine allgemeinverbindliche politische Kultur entwickelt und eine soziale Identifikation lediglich mit dem nächsten Bezugsumfeld wie Familie und Freundeskreis besteht. Hier übergreifende Interessenfindung und -wahrnehmung eines sozialen Bereichs organisiert durchsetzen zu können, stellt bereits eine weitere Stufe im nation-building eines Entwicklungslandes dar.

Die ökonomische Basis eines stetigen Wirtschaftswachstums erfordert generell eine nationale Kapitalbildung unter Verzicht auf die konsumptive Verwendung des Einkommens, d. h. die begrenzten Ressourcen eines Entwicklungslandes müssen in den Investitionsgütersektor gelenkt werden. Diese erste Phase einer Industrialisierung geht demnach auf Kosten einer Verbesserung des Lebensstandards der Bevölkerung. Inwieweit es hier nun gelingt, die organisierte Arbeiterschaft an der Realisierung eines nationalen Entwicklungsplanes - soweit ein solcher überhaupt angesichts der bekannten dependencia-Problematik erfolgreich sein kann ${ }^{1}$ - einzubinden, bestimmt maßgebend die wirtschaftliche Zukunft des Landes. Verzicht auf Lohnforderungen zwecks Bildung eines nationalen Kapitalmittelfonds unter Substituierung ausländischen Kapitals für als allseits notwendig erachtete nationale Investitionen setzt allerdings, ausgehend von dem konträren Selbstverständnis einer jeden Gewerkschaft, die Erfüllung gewisser Mindestbedingungen voraus. Auf dem Hintergrund der besonderen Problematik der Entwicklungsländer vornehmlich Lateinamerikas formuliert D. die Aufgaben der Gewerkschaften in dieser Ausgangssituation wie folgt (S. 41-43):

Es müsse garantiert sein, daß die nationalen Ressourcen, die durch den Konsumverzicht der Arbeiterschaft frei werden, nicht von der Elite selbst konsumiert oder

1 Mauro Marini, Ruy: Subdesarrollo y revolución, 4 a ed. México 1972, S. 17 ff. 
in Fehlinvestitionen verplant werden. Die von der Bevölkerung zu tragenden Lasten müßten hierin als gerecht verteilt akzeptiert werden können, z. B. durch ein progressives Steuersystem, mit dem sich gewisse Sozialinvestitionen finanzieren lassen. Es müßte nach der ersten Phase einer Industrialisierung sichergestellt sein, daß schon zur Schaffung eines ausreichenden Binnenmarktes für die nationale Industrie die Reallöhne angehoben werden.

Eine Zusammenarbeit auf dieser Basis zwischen Gewerkschaften und der politischen Elite erscheint immer dann möglich, wenn sich die Gewerkschaftsführung voll mit dem nationalen Entwicklungsplan identifizieren, wenn sie auf diese Weise zugleich effizienter, nämlich mit der Hilfe der politischen Gruppen die Lebensbedingungen der Arbeiterschaft verbessern kann. Indessen zeigt sich nun gerade in Lateinamerika, daß die Gewerkschaftsführer nicht den sozialen Druck auf das bisherige System verstärken, sondern vielmehr die Aufgabe übernehmen, die Arbeiterschaft zu disziplinieren und zu kontrollieren².

Der Einfluß der Gewerkschaften auf das politische System in Mexiko ist historisch vorgegeben durch den während der Revolution 1915 abgeschlossenen Pakt zwischen den Führern der Casa del Obrero Mundial und General Obregón, dem Befehlshaber der obsiegenden Streitkräfte Carranzas: Für die Gestellung von Arbeiterbataillonen versprach Carranza eine progressive Arbeitsgesetzgebung. Nach der Niederwerfung der Bauernbewegungen (Arbeiter gegen Bauern) wurde dieses Versprechen formell durch die Verfassung von 1917 erfüllt, in die erstmalig soziale Rechte der Arbeitnehmer aufgenommen wurden, vgl. Art. 123. Gleichwohl verfolgte Carranza als Präsident eine gewerkschaftsfeindliche Politik und veranlaßte die Gründung einer Gewerkschaftszentrale, die Confederación Regional Obrera Mexicana (CROM), in der "kooperative“ Gewerkschaftsfunktionäre die Spitzenpositionen besetzen konnten (S. $60 \mathrm{ff}$.). D. schildert äußerst detailreich (S. 60-107) die Entwicklung der mexikanischen Gewerkschaftsbewegung in ihrem Zusammenwirken mit dem jeweiligen Präsidenten. Jener Überblick zeigt, daß die Gewerkschaftsführer zwar im Rahmen der Präsidentschaftskampagne vor allem personelle Forderungen anbringen können (der Gewerkschaftsführer Morones wurde 1924 in Personalunion zum Wirtschafts- und Arbeitsminister ernannt) und beim Sieg des von ihnen unterstützten Kandidaten jedwede Förderung ihrer Gewerkschaft (etwa gegen konkurrierende Gewerkschaften) erfahren. Sobald jedoch die Gewerkschaftsführer zu mächtig werden oder ihre Politik mit der des Präsidenten nicht mehr übereinstimmt, hat es noch jeder Präsident verstanden, durch Unterstützung der Konkurrenzgewerkschaft, Spaltung der bisherigen Gewerkschaftsspitze, Verhinderung von Beitragserhebungen bei Staatsbediensteten und schließlich durch reine Repression seine eigene, meist nicht sehr arbeitnehmerfreundliche Politik durchzusetzen. Am Beispiel der einst geförderten, dann aber verfolgten CROM unter den Präsidenten Carranza, Obregón und Calles zeigte sich die Funktion einer regierungsabhängigen Gewerkschaftszentrale (S. 73): Die sozialpolitischen Forderungen der Arbeiterschaft sind niedrig zu halten, und gleichzeitig ist die Zustimmung der Arbeiter zur neuen Herrschaftselite als wahre Vollstreckerin der Revolution zu manifestieren. Erst unter dem Präsidenten Cárdenas (1934-1940) konnten sozialpolitische Fortschritte in der Sache erreicht werden. Zuvor mußte er sich jedoch mit Hilfe ihn unterstützender Gewerkschafts-

2 Millen, Bruce H.: The Political Role of Labor in Developing Countries, Washington 1963, S. 88. 
gruppen gegen die alte, ihm als "Linken" mißtrauisch gegenüberstehende CROMFührung durchsetzen (S. 79-82). Es lag in der Natur dieser miteinander sich verquickenden politischen und innergewerkschaftlichen Kämpfe, daß sich die Cárdenas unterstützenden Gewerkschaften 1936 zu einer neuen Zentrale, der Confederación de Trabajadores de México (CTM), zusammenschlossen, die in ihrer Verbindung zum Präsidenten alle übrigen Gewerkschaften übertrumpfen konnte und seither die herrschende Schicht Mexikos in ihrer jeweiligen politischen Ausrichtung (z. B. eine durchaus konservative unter Präsident Avila Camacho, 1940-1946)' absichert. Zur Abrundung dieser wechselseitigen Verflechtungen von Interessen und zwischen Personen gehört, daß die regierungsabhängigen Gewerkschaften im sog. sector obrero Mitglieder der Staatspartei Partido Revolucionario Institutional sind: Wer Mitglied dieser Gewerkschaften ist, ist automatisch Mitglied der Partei. Entsprechend sind die Gewerkschaften finanziell von der Regierung abhängig (S. 131/2), denn das Beitragsaufkommen ist nur gering, obwohl seit 1970 der Beitrag unmittelbar vom Lohn abgezogen und an die Gewerkschaften abgeführt wird, Art. 110 Abs. 6 Ley Federal del Trabajo.

D. schildert bis in die jüngste Zeit (1973) die personale Integration der Gewerkschaftsführer in die politische Elite Mexikos, die „Familia Revolucionaria“ (S. 220 ff.). Konflikte innerhalb dieser Machtelite werden regelmäßig intern ausgetragen (S. 233 f., nachgezeichnet am Beispiel der Postenjagd zwischen CTM-Funktionär und PRI-Politiker).

Aus dieser Zugehörigkeit zur Machtelite folgt, daß auch Gewerkschaftsführer Angriffe oppositioneller Gruppen jeder Art auf das System abwehren müssen. Die Behandlung sozialer Fragen durch den Erzbischof von Cuernavaca im Bundesstaat Morelos wurde von der CTM-Führung 1972 als Einmischung in Gewerkschaftsangelegenheiten angeprangert. Die Beschuldigungen gegenüber dem Erzbischof gingen soweit, ihn der Unterhaltung von Lagern für die Guerillaausbildung zu bezichtigen. Schließlich wurde an einem Sonntag eine Demonstration veranstaltet, so daß alle Messen und sonstigen religiösen Feiern ausfallen mußten (S. $243 \mathrm{ff}$.).

D. hat mit seiner Arbeit ausführlich nachgewiesen, daß in der oligarchischen Gesellschaftsstruktur Mexikos wohl die Gewerkschaftsführer als Teil der herrschenden Elite ihren Platz haben, die unterprivilegierte Position der Arbeiterschaft jedoch nachhaltig noch nicht verbessert ist. D. sieht allerdings in Übereinstimmung mit vielen anderen Beobachtern die Chance, daß sich Mexiko zu einem Sozialstaat moderner Prägung entwickelt, da sein Gesellschaftssystem so flexibel ist, Aufsteigern den sozioökonomischen Aufstieg und die Partizipation am Wirtschaftsaufschwung zu ermöglichen.

Gerhard Scheffler

\section{Leopold ZeA}

\section{Positivism in Mexico}

University of Texas Press, Austin \& London 1974, S. 241, XXIII (englische Ausgabe von: El Positivismo en México, México 1968).

Als am 19. Juni 1867 Kaiser Maximilian in Querétaro unter den Schüssen des Hinrichtungskommandos starb, hatte der politische Liberalismus unter Benito Juárez endgültig in Mexiko über die konservativen und klerikalen Kräfte der Ver- 